\title{
Stem cells, small RNAs, and self-renewal
}

\author{
Haifan $\operatorname{Lin}^{1}$ \\ ${ }^{I}$ Yale Stem Cell Center, Yale University School of Medicine, New Haven, CT 06520
}

The hallmark of stem cells is their ability to self-renew meanwhile capable of producing numerous differentiated daughter cells. Previously, we identified the ARGONUATE/PIWI protein family to be essential for stem cell selfrenewal in diverse organisms. This protein family can be divided into ARGONUATE and PIWI subfamilies. Recently, we and others discovered that the PIWI subfamily proteins interact with a novel class of non-coding small RNAs that we named PIWI-interacting RNAs (piRNAs) (Lin H, Science, 2007). There are at least 60000 species of piRNAs. They are mostly 26 32 nucleotides in length, and are abundantly expressed during spermatogenesis. Individual piRNAs frequently correspond to intergenic and repetitive sequences. In addition, a small number of piRNAs correspond to exonic and intronic sequences. This broad genomic distribution of piRNAs implicates their potential involvement in diverse mechanisms of gene regulation during spermatogenesis. Our latest work suggests that PIWI subfamily proteins and specific piRNAs are involved in epigenetic programming as well as the regulation of mRNA stability and, possibly, translation. Such regulations are involved in controlling the self-renewing division of germline stem cells and other gametogenic events in Drosophila and mammals.

Cell Research (2008) 18:s9. doi: 10.1038/cr.2008.99; published online 4 August 2008

Correspondence: Haifan Lin

E-mail: haifan.lin@yale.edu

Haifan Lin, PhD, Professor and Director of the Yale University Stem Cell Center. Dr. Lin work is focused on the self-renewing mechanism of Drosophila and mouse germline stem cells as well as human embryonic stem cells. In addition, Dr Lin studies germline development and cancers related to the malignant proliferation of stem cells. Dr Lin received his BS degree from Fudan University, and his PhD degree from Cornell University. Following his postdoctoral research at the Carnegie Institution of Washington, he joined the faculty of Duke University Medical School in 1994, where he rose to the rank of Full Professor. He co-founded and co-directed the Duke Stem Cell Research Program. Dr Lin moved to Yale

\begin{abstract}
in 2006 to establish the Yale Stem Cell Center. Dr Lin received numerous awards and honors, including the American Cancer Society Junior Faculty Research Award (1996), the March of Dimes Basil O onnor Scholar Research Award (1996), the David and Lucile Packard Fellowship for Science and Engineering (1996), Member of the Connecticut Academy of Science and Engineering (2007-), and the G Harold and Leila Y Mathers Award (2007). He has served on the NIH study sections (1998-2005), the International Society for Stem Cell Research (2002-), the Editorial Boards of Cell Stem Cells (2007-) and Stem Cells (2005-), and will be a Featured Editor for Nature Reports Stem Cells (2007). He has also served on the Scientific Advisory Boards of several leading research institutions and biotech companies, among other extramural activities.
\end{abstract}

